\title{
基于心理生理学视角的共情研究: 方法与特点
}

任巧悦 ${ }^{1,2}$, 孙元永 ${ }^{3}$, 吕雪靖 ${ }^{1,2^{*}}$, 黄超 ${ }^{4}$, 胡理 ${ }^{1,2^{*}}$

1. 中国科学院心理健康重点实验室(中国科学院心理研究所), 北京 100101;

2. 中国科学院大学心理学系, 北京 100049 ;

3. 辽宁师范大学脑与认知神经科学研究中心, 大连 116029;

4. 首都医科大学医学人文学院医学心理学学系, 北京 100069

*联系人, E-mail: luxj@psych.ac.cn; huli@psych.ac.cn

2019-05-05 收稿, 2019-05-16 修回, 2019-05-17 接受, 2019-07-17 网络版发表

国家自然科学基金(31671141，31701000，31822025)、中国科学院“科研信息化应用工程”(XXH13506-306)、中国科学院心理研究所科研启动项 目(Y6CX021008)和中国科学院心理健康重点实验室经费(KLMH2018ZG02)资助

摘要 共情使个体能够理解并分享他人的感受, 对人际交往有重要意义.近年来, 基于量表、行为范式、生理信号 等多个层面的共情研究方法有了长足进展, 为研究不同维度的共情(如情绪共情、认知共情)的机制提供了多方面 的信息. 然而, 由于“共情”缺乏统一定义, 研究者根据各自理解开发了多样化的研究方法, 这阻碍了对现有共情研 究结果的比较和整合. 本文基于情绪共情和认知共情两个维度对量表、行为范式、生理信号3个层面的常用共情 研究方法进行了分类总结. 首先, 本文围绕共情概念、测量维度等方面简要介绍若干影响力较大的共情量表, 如 情绪倾向量表、霍根共情量表、人际反应指针量表等. 其次, 本文总结并比较针对不同共情维度的常用实验材料 及行为范式的特点. 再次, 本文系统介绍如何在共情研究中利用面部肌电、脑电、脑磁和磁共振成像等方法提取 共情相关的生理信号并分析其特点. 最后, 本文综合对比不同层面共情研究方法的特点, 并建议研究人员根据研 究需要谨慎选择研究方法. 此外, 针对当前共情研究存在的可比性问题和生态效度问题, 建议对不同研究结果进 行比较和整合时特别注意共情概念是否一致; 未来研究需开发更具生态效度的行为范式, 重点探究共情的动态加 工过程.

关键词 共情, 情绪共情, 认知共情, 量表, 行为范式, 生理信号

共情(empathy)一词最早由英国心理学家Edward Titchener于1909年从德语翻译而来, 意为“感受 到”(feeling into) ${ }^{[1]}$. 迄今, 共情研究已有上百年的历史. 一般来讲, 共情指个体感知他人心理状态的敏感性, 包 括被他人状态感染、体验到与他人相同的状态、评估 状态产生的原因、理解他人的想法等一系列心理过 程 ${ }^{[2]}$. 根据所涉及的心理加工过程的不同, 共情可大致 分为情绪共情(emotional empathy)和认知共情(cognitive empathy $)^{[3]}$. 情绪共情指替代性地体验他人的心理
状态, 是较原始、初级的共情维度, 是刺激驱动的自动 化的过程; 认知共情指外显地理解他人的心理状态及 其原因, 涉及更多的高级认知活动. 目前, 研究者利用 量表、行为范式、生理信号记录等方法从多个层面对 共情的不同维度进行了研究(图1).

由于共情的定义尚未统一, 不同研究者常使用“共 情”一词指代共情的不同维度并采用不同的专业术语 描述相似的共情维度 ${ }^{[3]}$. 国内共情研究也面临共情相关 术语、定义不统一的问题 ${ }^{[\sim 6]}$. 例如, “empathy”的中文 


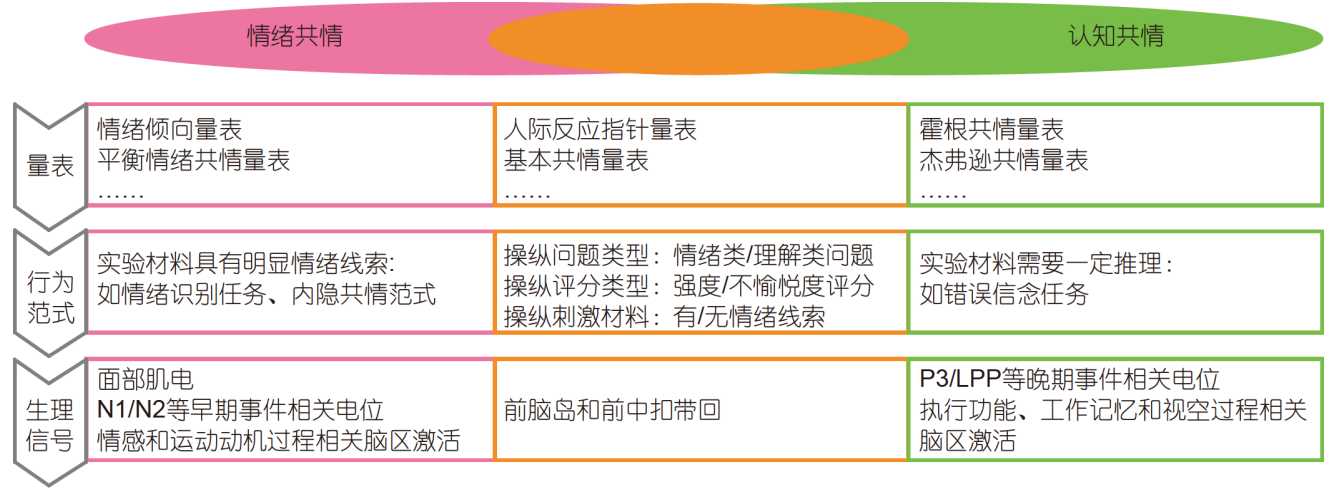

图 1 (网络版彩色)常用的共情研究方法和指标

Figure 1 (Color online) Common questionnaires, behavioral paradigms, and physiological signals in empathy research

译名除了“共情”, 还包括“移情”、“同感”、“共 感”、“同理心”等 ${ }^{[4,7]}$. 此外, 由于同情(sympathy)在词源 和翻译上和共情近似，现有中文文献中还存在“共情” 和“同情”使用混乱的情况 ${ }^{[6]}$. 研究者对共情概念的不同 理解使得各个研究层面产生了多样化的研究方法, 给 共情研究方法的选择和研究结果的解释和整合带来了 巨大的阻碍.

为了有效整合不同层面的研究, 更加深人地理解 共情及其机制, 本文旨在系统总结近年来共情在量 表、行为范式、生理信号 3 个层面的研究成果. 具体而 言, 本文从情绪共情和认知共情两个维度出发, 对影响 力较大的共情量表、常用行为范式及实验材料和利用 面部肌电(electromyography, EMG)、脑电(electroencephalography, EEG)、脑磁(magnetoencephalography, MEG)和磁共振成像(magnetic resonance imaging, MRI) 提取的共情相关生理信号进行总结. 最后, 本文综合对 比各类研究方法的优劣, 指出目前共情研究的可比性 问题和生态效度问题, 并对未来共情研究方法的改进 提出了建议.

\section{1 共情研究常用量表简介}

基于共情的不同概念及维度, 迄今研究者们已编 制了数十个共情量表(常用共情量表见表1). 这些量表 可大致分为测量情绪共情、测量认知共情以及同时测 量情绪共情和认知共情这 3 类. 标准化的量表有助于研 究者方便、快捷地对个体的共情能力进行评估.

\section{1 测量情绪共情的量表}

Mehrabian和Epstein编制的情绪倾向量表(emotional empathic tendency scale, EETS, 又称questionnaire measure of emotional empathy, QMEE) ${ }^{[8]}$ 是目前使用最 广泛的测量情绪共情的量表. 在该量表中, 情绪共情指 “对他人情绪体验的反应” ${ }^{[8]}$. QMEE编制基于的假设是: 情绪共情水平更高的人对他人的情绪感受有更强的反 应性, 即在注意到他人的痛苦时, 具备高情绪共情水平 的人更可能有帮助行为而非攻击行为. 该量表的 33 个 条目涉及情绪感染敏感性、对陌生人情绪的理解、极 端情绪反应、积极情绪感动的倾向、消极情绪感动的 倾向、同情倾向、自愿接触不幸他人的倾向 7 个因子. 例如，情绪感染敏感性的代表性题目是“我周围的人对 我的情绪有很大的影响”, 而自愿接触不幸他人的倾向 的代表性题目是“我宁愿做一名社会工作者而不是在 职业培训中心工作”。然而, 该量表的作者后来指出, QMEE分数很大程度上反映的是一般性的情绪唤醒 (emotional arousability)而非共情 ${ }^{[9]}$. 于是, 该作者编制 了全新的“平衡情绪共情量表”(balanced emotional empathy scale, BEES $)^{[10]}$. BEES聚焦于测量个体“替代性地 感受他人情绪的能力”, 包含“令人悲伤的电影结束几 小时后仍困扰着我” 和“我不能对那些因为自己而导致 痛苦的人感到悲伤”等条目.

常见的情绪共情量表还包括多维度情绪共情量表 (multidimensional emotional empathy scale, MDEES) ${ }^{[11]}$ 、情绪感染量表(emotional contagion scale, $\mathrm{ECS})^{[12]}$ 等. 这些量表所测量的情绪共情在概念上均有 细微差别, 如MDEES涉及情绪感染(emotional contagion)、共情痛苦(empathic suffering)等多个维度, 而 ECS仅聚焦情绪感染这一个维度.

\section{2 测量认知共情的量表}

霍根共情量表(Hogan empathy scale, HES $)^{[13]}$ 是测 
表 1 常用共情量表 ${ }^{\text {a) }}$

Table 1 Frequently-used empathy scales ${ }^{\text {a) }}$

\begin{tabular}{|c|c|c|c|c|c|c|}
\hline 缩写 & 量表全称 & 中文译名 & 施测对象 & 测量维度 & $\begin{array}{l}\text { 来源 } \\
\text { 文献 }\end{array}$ & 引用量 \\
\hline IRI & interpersonal reactivity index & 人际反应指针量表 & 通用 & 情绪共情, 认知共情 & {$[20]$} & 7322\# \\
\hline AQ & autism quotient & 自闭症商数量表 & 自闭症患者 & 1 & {$[38]$} & $2077 *$ \\
\hline EQ & empathy quotient & 共情商数问卷 & 成人 & 情绪共情, 认知共情 & {$[15]$} & $1358^{*}$ \\
\hline $\begin{array}{l}\text { EETS } \\
\text { (QMEE) }\end{array}$ & $\begin{array}{l}\text { emotional empathic } \\
\text { tendency scale } \\
\text { (questionnaire measure of } \\
\text { emotional empathy) }\end{array}$ & 情绪倾向量表 & 通用 & 情绪共情 & {$[8]$} & $1062 *$ \\
\hline HES & Hogan empathy scale & 霍根共情量表 & 通用 & 认知共情 & [13] & $665^{*}$ \\
\hline CATS & $\begin{array}{l}\text { child and adolescent } \\
\text { temperament scale }\end{array}$ & 儿童青少年气质量表 & 儿童、青少年 & 1 & [39] & $468^{*}$ \\
\hline BES & basic empathy scale & 基本共情量表 & 青少年 & 情绪共情, 认知共情 & [32] & $311^{*}$ \\
\hline JSE & Jefferson scale of empathy & 杰弗逊共情量表 & $\begin{array}{c}\text { 医护工作者、 } \\
\text { 学生 }\end{array}$ & 认知共情 & {$[16]$} & $310^{*}$ \\
\hline ECS & emotional contagion scale & 情绪感染量表 & 通用 & 情绪感染 & {$[12]$} & $515 \#$ \\
\hline РPРB & $\begin{array}{l}\text { Penner prosocial } \\
\text { personality battery }\end{array}$ & 佩纳亲社会人格量表 & 通用 & 1 & {$[40]$} & $471 \#$ \\
\hline TEQ & Toronto empathy measure & 多伦多共情量表 & 通用 & / & {$[41]$} & $126^{*}$ \\
\hline SEE & scale of ethnocultural empathy & 民族文化共情量表 & 通用 & l & {$[42]$} & $346 \#$ \\
\hline CARE & $\begin{array}{l}\text { consultation and relational } \\
\text { empathy measure }\end{array}$ & 咨询和关系共情量表 & 通用 & / & {$[43]$} & $209^{*}$ \\
\hline BEES & $\begin{array}{l}\text { balanced emotional } \\
\text { empathy scale }\end{array}$ & 平衡情绪共情量表 & 通用 & 情绪共情 & {$[10]$} & $263 \#$ \\
\hline QCAE & $\begin{array}{l}\text { questionnaire of cognitive and } \\
\text { affective empathy }\end{array}$ & 认知和情绪共情问卷 & 通用 & 情绪共情, 认知共情 & {$[34]$} & $257 \#$ \\
\hline GEM & Griffith empathy measure & 格里菲斯共情量表 & 家长 & 情绪共情, 认知共情 & {$[36]$} & $751^{*}$ \\
\hline FTS & feeling and thinking scale & 感受和思维量表 & 儿童 & 情绪共情, 认知共情 & {$[35]$} & $70 \#$ \\
\hline PACES & $\begin{array}{l}\text { parent affective and cognitive } \\
\text { empathy scale }\end{array}$ & $\begin{array}{c}\text { 父母情绪和认知 } \\
\text { 共情量表 }\end{array}$ & 家长 & $\begin{array}{c}\text { 情绪共情, 认知共情, } \\
\text { 行为共情 }\end{array}$ & {$[44]$} & $22 *$ \\
\hline AMES & $\begin{array}{l}\text { adolescent measure of empathy } \\
\text { and sympathy }\end{array}$ & $\begin{array}{l}\text { 青少年共情和 } \\
\text { 同情量表 }\end{array}$ & 青少年 & $\begin{array}{c}\text { 情绪共情, 认知共情, } \\
\text { 同情 }\end{array}$ & {$[45]$} & $41 \#$ \\
\hline EmQue & empathy questionnaire & 共情问卷 & 家长 & l & {$[46]$} & $31 *$ \\
\hline EPS & empathy for pain scale & 疼痛共情量表 & 通用 & l & [47] & $10^{*}$ \\
\hline MDEES & $\begin{array}{l}\text { multidimensional emotional } \\
\text { empathy scale }\end{array}$ & 多维度情绪共情量表 & 青少年、成人 & 情绪共情 & {$[11]$} & $11 \#$ \\
\hline ESE & $\begin{array}{l}\text { emotion specific empathy } \\
\text { questionnaire }\end{array}$ & 情绪特异性共情问卷 & 通用 & 情绪共情, 认知共情 & [48] & $10^{*}$ \\
\hline EES & emotional empathy scale & 情绪共情量表 & 通用 & 情绪共情 & [49] & $13 \#$ \\
\hline
\end{tabular}

a) 引用量来自Web of Science $\left(^{*}\right)$ 和谷歌学术(\#, 适用于无法获取Web of Science数据的情况), 截至2019年1月10日; “测量维度”一列仅表示 按照情绪共情和认知共情角度对量表维度的归类, 不表示量表的确切维度; “”指该量表将共情视为整体且无法归类

量认知共情的经典量表之一. 在该量表中，共情指“在 没有真正体验到他人感受的情况下，对他人的状况或 心态的想象和理解”。该量表共有 64 个条目，涉及社会 自信、温和、敏感性和不一致性 4 个因子. 然而, 有研
究指出, 该量表的信效度较低, 不建议将其作为测量共 情的工具继续使用 ${ }^{[14]}$. 还有研究者认为, HES中的部分 条目(如“能否意识到要给他人留下好印象”)测量的是 社会技能而非认知共情 ${ }^{[15]}$. 
另一个测量认知共情的量表是杰弗逊共情量表 (Jefferson scale of empathy, JSE) ${ }^{[16]}$. 该量表关注医护领 域工作者的共情, 已经有超过45种语言的翻译版本, 并 在超过 70 个国家使用。该量表作者将临床情境中的共 情定义为“理解(而非感受)病人的疼痛、痛苦、经历和 担心”，相对于共情的情绪维度，该定义主要强调共情 的认知维度 ${ }^{[17]}$. JSE共有 3 个版本, 内容相似, 仅措辞略 有不同，分别适用于测量医生、医学生和除医学外其 他医疗相关专业学生的共情 ${ }^{[17]}$. 因素分析结果表明, JSE的条目涉及观点采择、同情关怀、站在患者的角 度 3 个因子 ${ }^{[17]}$. 其中, 观点采择强调对病人观点的看法; 同情关怀强调对病人经历的理解；站在患者的角度则 强调在护理病人时不忽视病人的情绪 ${ }^{[18]}$. 同情关怀常 被误认为测量的是共情的情绪维度，但JSE的作者曾指 出，JSE基于共情的认知维度编制，对其进行量表结构 分析不应出现、事实上也的确未出现情绪维度 ${ }^{[19]}$.

\section{3 测量情绪共情和认知共情的量表}

共情是一个多维度的概念，同时考虑共情的情绪 维度和认知维度才能全面衡量人们的共情能力 ${ }^{[20]}$. 因 此，更多的共情量表被设计为能够同时测量情绪共情 和认知共情.

Davis编制的人际反应指针量表(interpersonal reactivity index, IRI) 是迄今为止引用率最高的共情量表 ${ }^{[20]}$ IRI包括共情关注(empathic concern，EC)、个人痛苦 (personal distress，PD)、观点采择(perspective taking, PT)和想象(fantasy，FS)4个分量表. 其中，EC评估对他 人情绪的关心，PD评估对他人痛苦产生的负性感受， PT评估在认知上理解他人想法的倾向，而FS评估对书 籍、电影或戏剧中的角色的情绪认同. Davis认为 $\mathrm{EC}$ 和 PD测量的是情绪共情维度, PT和FS测量的是认知共情 维度 ${ }^{[20]}$. 但是也有研究者质疑FS和PD评估的分别是想 象力和情绪自我控制的能力, 与共情无关 ${ }^{[15]}$. 值得注意 的是，IRI中的“观点采择”和JSE中的“观点采择”虽然名 称一样, 所评估的共情维度并不一致 ${ }^{[19]}$. 例如, 对大样 本的IRI和JSE分数的分析结果表明，两个量表的“观点 采择”分数没有显著相关 ${ }^{[21]}$. 中文版人际反应指针量表 (interpersonal reactivity index-C, IRI-C)(繁体中文版)最 初于1987年由中国台湾学者詹志禹翻译及修订, 由原 量表的 28 个条目删减至 22 个条目，仍包含上述 4 个维 度 ${ }^{[22]}$. 后续大陆学者将其转换为简体中文版, 并证实了 该量表在健康人、精神病患者、临床实习生等不同人
群中均有良好的信效度 ${ }^{[23,24]}$. 还有一些学者考察了自 主翻译的IRI在不同群体(如护理专业学生 ${ }^{[25]}$ 、非护理 专业学生 ${ }^{[26-28]}$ 、中小学教师 $\left.{ }^{[29]}\right)$ 中的信效度, 修订中有 个别题目的删减和维度的变化，但均证实了相应翻译 版IRI在中国文化背景下的适用性. 例如, Siu和Shek ${ }^{[26]}$ 把原量表中的PT和EC合并为一个分量表，并删除了 6 个题目, 得到了适用于香港人群的繁体中文版IRI.

近年来，研究者还开发了能同时测量情绪共情和 认知共情的其他量表，呈现出共情定义精确化和适用 范围明确化的发展趋势. Baron-Cohen和Wheelwright将 共情定义为“将心理状态归因于他人/动物，并且对他 人/动物的心理状态做出恰当的情绪反应的动力或能 力”并据此编制了共情商数量表(empathy quotient, $\mathrm{EQ})^{[15]}$. 该量表包含认知共情、情绪反应和社会技能3 个维度，但社会技能维度测量的是在特定场合下的行 为举止，与共情无关 ${ }^{[30]}$. 除了适用于智商正常的成年 人, 该量表还常被用于孤独症群体的共情研究 ${ }^{[15]}$. 该量 表中文版的内部一致性系数为 0.86 , 重测信度为 $0.82^{[31]}$. Jolliffe和Farrington ${ }^{[32]}$ 编制的基本共情量表(basic empathy scale，BES)将共情定义为“理解和分享他人的情绪 状态或处境”。该量表基于高兴、生气、悲伤和恐惧4 种基本情绪对情绪共情和认知共情进行评估; 在具体 条目中避免引入“同情”这一混淆概念; 避免了以往量 表编制过程中常见的目标群体和实际测量群体不一致 的问题 ${ }^{[32]}$. 该量表中文版的内部一致性系数为 0.77 , 重 测信度为 $0.70^{[33]}$. 认知和情绪共情量表 (questionnaire of cognitive and affective empathy, QCAE)则是第一个通过 网络在线施测的共情量表，它不仅区分了共情与同情, 还区分了认知共情与心理理论(theory of mind)，并强 调情绪共情中自我和他人的区分 ${ }^{[34]}$. 为弥补儿童共情 测量的空白，有研究者将著名的IRI改编成了感受和思 维量表(feeling and thinking scale, FTS) ${ }^{[35]}$. 此外, Dadds等人 ${ }^{[36]}$ 开发了针对儿童共情的父母报告量表 格里菲斯共情量表(Griffith empathy measure, GEM).Zhang等人 ${ }^{[37]}$ 将该量表翻译成中文，并修改了 个别条目内容. 值得注意的是, 该中文版格里菲斯共情 量表为自陈量表，且仅被证明在大学生样本中有较高 的信效度 ${ }^{[37]}$

\section{2 共情研究经典行为范式}

共情不仅被视为一种相对稳定的特质或能力，还 被视为一种具备可变性(variability)的状态，受到认知 
负荷 ${ }^{[50]}$ 和情绪 ${ }^{[51]}$ 等因素的影响. 基于这种界定, 研究者 通常设计具体的行为范式以测量被试的共情水平. 共 情研究常用行为范式的基本逻辑如下：给被试呈现直 接或间接反映他人情绪、心理状态或所处情境的实验 材料，然后要求被试对实验材料所诱发的自身情绪或 心理状态进行评分，或者对实验材料中他人的情绪或 心理状态进行评分.

常用共情实验材料包括图片 ${ }^{[52]}$ 、视频 ${ }^{[33]}$ 、符号/ 线索 ${ }^{[54]}$ 、叙述性文字 ${ }^{[55]}$ 等. 实验材料有不同的呈现方 式: 以第一视角呈现刺激直接诱发共情, 有利于被试更 好地“感同身受”; 以第三视角呈现刺激, 利用指导语对 被试与被共情者的关系进行操纵, 要求被试想象实验 材料所呈现的是陌生人、朋友或恋人等的遭遇，有利 于被试以旁观者的角度对“他人”进行共情，探究人际 关系或熟悉度对共情的影响 ${ }^{[56]}$. 操纵视角还有助于研 究者对比自身表征(self-representation)和共情时对他人 表征(other-representation)的异同 ${ }^{[57]}$.

参照常用共情量表分类，这些行为范式也可大致 分为测量情绪共情、测量认知共情以及同时测量情绪 共情和认知共情 3 类.

\section{1 情绪共情行为范式}

基于情绪共情的定义，常用行为范式往往从情绪 诱发、情绪识别等角度探索个体的情绪共情能力, 并 采用简单明了且具有明显情绪线索的实验材料, 减少 甚至排除推理等高级认知活动的干扰. 如在疼痛情绪 共情行为范式中, 实验材料通常是含有明显伤害性、 威胁性内容(如刀割手指)的图片 ${ }^{[52]}$, 或痛苦面孔表情 的图片 ${ }^{[58]}$, 甚至是动态的情绪性视频(如一个人讲述不 幸的处境 $)^{[53]}$ ，以直接诱发被试的情绪唤醒，然后要求 被试对疼痛程度进行评估. 又如在情绪识别任务 (emotion recognition task)中, 被试被要求对不同的面部表情 所表达的情绪类型进行判断. 这种范式测得的情绪识 别能力被认为是情绪共情的重要组成部分, 因此个体 的任务表现能够反映其情绪共情 ${ }^{[59]}$.

情绪唤醒的直接诱发和情绪识别的主观判断都反 映了情绪共情的现象学部分, 聚焦主观感受和判断, 但 不可避免地存在报告偏差. 内隐共情范式(implicit empathy paradigms)将被试在进行共情无关任务时受共情 材料的干扰程度作为衡量共情的间接指标，能有效克 服主观报告偏差的问题. 例如, 在疼痛共情内隐范式 中，虽然给被试呈现的是疼痛或非疼痛的身体部位的
图片, 但实验并不要求被试对图片中的疼痛信息作判 断或者识别, 而是对其他信息进行又快又好地判断, 如 判断图片上呈现的手是左手还是右手 ${ }^{[60]}$. 这种范式的 前提假设是: 展示他人受伤的图片会唤醒被试的共情 反应, 如共情痛苦(empathic distress), 从而干扰其任务 表现, 如增加反应时和降低正确率. 因此, 被试的情绪 共情越强，其对疼痛无关信息的判断越容易受到干 扰 ${ }^{[61]}$.

\section{2 认知共情行为范式}

基于认知共情的定义, 测量认知共情的行为范式 重点考察被试对他人心理状态的推理能力. 因此, 认知 共情任务的实验材料往往缺乏直接、明确的情绪线索, 如叙述犯罪受罚的文字材料 ${ }^{[55]}$ 、呈现威胁情境的视 频 ${ }^{[62]}$ 等. 被试需要调动更多的高级认知过程对他人的 心理状态进行理解. 常用的认知共情行为范式还有漫 画形式的二阶错误信念任务(second-order false belief task), 该范式着重于考察个体对多人情境的理解和对 第三人想法的推理能力 ${ }^{[59]}$. 实验材料(简笔漫画)中缺 乏直接的情绪状态线索, 即未呈现漫画人物的表情, 被 试需要理解漫画中的多人情境并推理人物的心理状态, 任务的完成水平即为认知共情的指标 ${ }^{[63]}$. 这类任务的 难度容易调节, 因此也被广泛应用于儿童共情研究 ${ }^{[64]}$. 此外, 有研究者认为认知共情和情感心理理论(affective theory of mind)均涉及个体对他人情绪的理解 ${ }^{[65]}$, 因此 可以利用情感心理理论任务来测量认知共情. 例如, 有 研究要求被试观看一个名叫“Yoni”的卡通人物的眼神 和面部表情并据此判断“Yoni”对他人或事物所持有的 情绪. 由于涉及一定的推理加工, 被试的任务表现能够 反映其认知共情能力 ${ }^{[66]}$.

\section{3 同时测量认知共情和情绪共情的行为范式}

除了单独的情绪共情任务和认知共情任务外, 研 究者还开发了能同时考察情绪共情和认知共情的行为 范式. 例如, 多维共情测验(multifaceted empathy test, MET)以图片为实验材料, 其中一半图片呈现的是人物 在特定情境下的情绪性(如伤心、害怕或痛苦)经历, 另 一半图片仅呈现相应的情境而没有人物 ${ }^{[67]}$. 被试观看 图片后, 需要回答一系列的问题. 对于纯情境图片, 被 试只需要对唤醒度进行评分. 对人物情境图片, 被试需 要先推断图片中人物的心理状态, 得到正确答案的反 馈后, 再对图片的唤醒度进行评分, 最后对共情关注程 
度进行评分. 在MET中, 唤醒度评分是对情绪共情的内 隐测量, 共情关注评分是对情绪共情的外显测量, 对人 物心理状态的推断则是对认知共情的测量. 纯情境图 片和人物情境图片的设置还便于研究者区分被试对非 社会性刺激(情境)的情绪反应和对人物的共情反应 ${ }^{[67]}$ 之后, 有研究者基于MET开发了类似的行为范式, 用被 试观看图片后对图中人物感受的推断来衡量其认知共 情，用被试对图片引发的自身感受的评分来衡量其情 绪共情 ${ }^{[68]}$.

此外, 在某些疼痛共情研究中, 研究者认为被试对 他人疼痛强度的评分涉及对他人疼痛躯体感觉的推理, 反映的是认知共情; 而被试对他人痛苦引起自身的不 愉悦感受的评分涉及替代性的情绪体验，反映的是情 绪共情 ${ }^{[69]}$. 还有研究者通过操纵行为范式中情绪线索 的有无来分离情绪共情和认知共情，对负性场景(如人 际攻击情境)中人物的共情被视作对他人的情绪状态 的知觉，因此归为情绪共情; 而对中性场景(如日常非 感情活动)中人物的共情被视作对他人感受或意图的 推断, 因此归为认知共情 ${ }^{[70]}$.

总之，目前已有研究都是根据各自的共情定义试 图分离情绪共情和认知共情, 尚无统一的分离范式. 但 是, 分类的核心原则比较一致, 即直接的情绪分享属于 情绪共情, 而涉及推理过程的属于认知共情.

\section{3 共情相关神经科学研究方法}

行为范式能够采集被试的主观评分，而辅助的电 生理技术(如EMG，EEG，MEG等)和脑成像技术(如 $\mathrm{MRI}$ )能进一步提供共情的客观生理和脑活动水平指 标. 这些技术在共情研究中的应用极大促进了研究者 对共情现象背后的神经加工过程的了解.

\section{1 面部肌电}

运动模仿是共情的核心成分 ${ }^{[2]}$. 研究对象为人类 ${ }^{[71]}$ 和灵长类动物 ${ }^{[72]}$ 的都发现，观察者会对被共情者进行 快速的面部表情模仿(facial mimicry). 研究者可以通过 测量被试在面部模仿过程中骨骼肌收缩时产生的电位 (即面部肌电), 量化其面部模仿情况 ${ }^{[73]}$. 其中, 能反映共 情的面部表情主要涉及皱眉肌、颧肌、上唇提肌、眼 轮匝肌等面部肌肉的活动 ${ }^{[74]}$.

除面部表情模仿外，个体对他人面部表情的反应 灵敏度和面部反应的电位强度也能有效反映其情绪共 情能力. 研究表明, 情绪共情得分高的个体对愤怒面孔
刺激会表现出更大的皱眉肌活动，对快乐面孔刺激会 表现出更大的颧肌的活动，而情绪共情得分低的个体 对两类面孔刺激反应无显著差异 ${ }^{[75,76]}$. 此外, 被试在观 看他人或动物处于负性情境中的实验材料时，其皱眉 肌的电位幅度与共情主观评分显著正相关，还与BEES 分数显著正相关 ${ }^{[77]}$.

虽然EMG时间精度高，能够检测视觉阈值以下的 面部反应，但是EMG信号中可能掺杂了对共情无关的 视觉刺激的肌电响应. 此外, 将电极贴附在被试面部也 可能增加其对面部表情的关注，从而导致面部反应夸 $大^{[74]}$. 最重要的是, 由于肌肉活动并不是共情特异性反 应, 且研究中往往只记录某些特定的面部肌肉, 因此 EMG结果往往需要结合其他反映共情的指标来解释.

\section{2 脑电、脑磁}

EEG信号也常作为一种评估被试在共情行为范式 中共情水平的客观指标 ${ }^{[78]}$. 以疼痛共情为例, 2008年的 一项开创性的疼痛共情事件相关电位(event related potentials, ERPs)研究表明, 相比非疼痛图片, 被试会在疼 痛图片呈现后约 $140 \mathrm{~ms}$ 于额叶区域诱发一个波幅更大 的ERP成分(即N1/N2), 并在疼痛图片呈现后约 $380 \mathrm{~ms}$ 于中心-顶叶区域诱发一个波幅更大的ERP成分(即P3/ LPP $)^{[79]}$. 此后, 大量研究结果支持了这一结论，即早期 的自动化的 $\mathrm{N} 1, \mathrm{~N} 2$ 和晚期的P 3 , LPP都与共情有关. 例 如, 疼痛共情范式诱发的早期ERP成分和晚期ERP成分 的波幅不仅与被试的特质共情分数(IRI或 $E Q$ 分数)显 著正相关 ${ }^{[80,81]}$, 还与其对他人的疼痛强度和不愉悦度 的主观评分正相关 ${ }^{[82,83]}$. 而且，当实验任务与疼痛共情 的加工无关时, 疼痛图片和中性图片引起的早期ERP 成分和晚期ERP成分的波幅差异均减小 ${ }^{[79,84]}$; 当实验 任务强调共情加工时，两类图片引起的早期ERP成分 和晚期ERP成分的波幅差异均增加 ${ }^{[85]}$. 这些ERP成分 甚至受到被共情者类型的影响. 例如, 相比对同种族个 体，被试对卡通 ${ }^{[79]}$ 、机器人 ${ }^{[86]}$ 或者其他种族个体 ${ }^{[87]}$ 共 情时产生的P2或P3的波幅更小.

但是，早期ERP成分和晚期ERP成分分别反映的是 共情加工的哪一具体过程还存在争议. 由于这两类成 分分别受到刺激本身的特点(如真实性)和认知评价(如 注意)的调节, 以往研究者普遍将 $\mathrm{N} 1$ 和 $\mathrm{N} 2$ 作为反映早期 的情绪分享过程的指标, 将P3 和LPP作为反映晚期的对 他人疼痛的认知评估过程的指标 ${ }^{[79,88,89]}$. 而最近的一项 基于 40 个疼痛共情ERP研究的元分析结果表明, 疼痛共 
情能够稳定诱发波幅更大的位于中部-顶叶的P3和LPP, 但是不一定能诱发波幅更大的 $\mathrm{N} 1$ 和 $\mathrm{N} 2{ }^{[90]}$. 该作者进一 步指出, 这可能是因为早期的 $\mathrm{N} 1$ 和 $\mathrm{N} 2$ 反映的是对刺激 的知觉加工, 并未涉及真正的共情反应. 而且, 由于多 种类型的情绪性刺激都能诱发这些早期ERP成分和晚 期ERP成分 ${ }^{[91]}$ ，因此这些成分很可能不是共情特异性 的, 而是反映一般性的知觉加工和厌恶加工 ${ }^{[90]}$.

除了ERP，特定的EEG节律也与共情水平相关. 如 在观看他人疼痛时会引起mu/alpha节律 $(8 \sim 13 \mathrm{~Hz})$ 抑制 程度的增强 ${ }^{[92,93]}$, 这种 $\mathrm{mu} / \mathrm{alpha}$ 节律的抑制源于感觉运 动皮层和初级体感皮层等共情早期加工脑区的激活， 因而能够反映疼痛共情的早期加工 ${ }^{[78]}$. 此外, 由于前额 叶的激活与情绪体验有关而与情绪知觉无关 ${ }^{[94]}$, 情绪 体验是情绪共情的核心加工过程，因此共情任务中个 体前额叶alpha不对称活动常被视为衡量被试情绪共情 的良好指标 ${ }^{[95]}$. 进一步研究发现, 被试在基线状态下 (即刺激呈现前)右侧前额叶alpha频段的不对称程度能 够预测其在任务中的共情关注评分，意味着该脑区活 动能反映被试对他人痛苦的敏感性 ${ }^{[96]}$.

共情涉及多个加工过程, 单个的EEG节律可能与 共情有关, 却不足以完整解释共情的脑机制. 研究者可 以利用MEG的高空间分辨率和时间分辨率的优势，探 索共情任务中跨越多个脑区多个频段的振荡信号的动 态关联. 有研究者探究不同年龄段个体共情的脑机制 的差别, 发现即使儿童和成人的疼痛共情评分近似, 但 从儿童到成人，共情脑活动从单一的alpha节律转变为 alpha, beta(14 30 Hz) 和gamma(30 100 Hz)多节律的高 效相互关联, 并从感觉运动脑区扩展到情绪共情脑网 络 ${ }^{[97]}$.

\section{3 磁共振成像}

MRI具有较高的空间分辨率，能够更加直观、精 细地刻画大脑的结构和功能. 通过分析个体大脑结构 像(如特定脑区的灰质体积)与共情特质之间的关系, 研 究者发现IRI中的EC分数与楔前叶(precuneus)、额下 回(inferior frontal gyrus)、前扣带回(anterior cingulate cortex, ACC)的灰质体积负相关; PD分数与躯体感觉皮 层(somatosensory cortex)的灰质体积负相关, 但与脑岛 (insula)的灰质体积正相关; PT分数与ACC的灰质体积 正相关; FS 分数与右背外侧前额叶皮层(dorsolateral prefrontal cortex, DLPFC)的灰质体积正相关 ${ }^{[98]}$. 对共 情的功能像研究发现, 情绪共情任务在更大程度上涉
及情绪和运动相关脑区的激活，如脑岛、ACC、丘 脑、杏仁核、梭状回、躯体感觉皮层、运动皮层和腹 内侧前额叶皮层(ventromedial prefrontal cortex， VMPFC); 认知共情任务在更大程度上涉及与执行功 能、工作记忆和视觉空间处理等相关脑区的激活，如 DLPFC、背内侧前额叶(dorsomedial prefrontal cortex)、颞上回(superior temporal gyrus, STG)、颞顶联 合区(temporoparietal junction，TPJ)、上顶叶(superior parietal lobule)和下顶叶(inferior parietal lobule, IPL) ${ }^{[2]}$.

此外, 利用功能磁共振成像(functional magnetic resonance imaging, fMRI)较高的空间分辨率, 研究者得以 发现同类共情范式所用的实验材料不同可能引起不同 的脑活动. 例如, 在疼痛共情行为范式中, 观看身体部 位处于疼痛情境下的图片在更大程度上涉及与行为理 解相关的脑区, 如IPL、腹侧运动前皮质(ventral premotor cortices); 观看表示他人情绪状态的抽象视觉信息在 更大程度上涉及与推断和表征自我和他人心理状态相

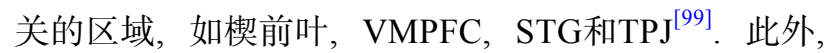
fMRI能够精确地呈现脑损伤患者的脑受损部位，便于 探究特定脑区损伤与共情的因果关系. 有研究表明, 躯 体感觉皮层的损伤会造成情绪共情而非认知共情的缺 陷，与之相反，VMPFC的损伤会导致认知共情而非情 绪共情的异常 ${ }^{[59]}$, 这给情绪共情和认知共情脑区的分 离进一步提供了证据.

需要注意的是, fMRI测量的是与某一心理认知过 程相关的大脑活动, 因而存在逆向推理(reverse inference)的问题 ${ }^{[100]}$ ，即无法证明这些脑区是共情特异性 的. 此外, fMRI结果的单个体素(voxel)涵盖了数千个的 神经元, 在功能上不同的神经元活动能产生相似的 fMRI激活图, 因此重叠的激活脑区不一定代表同样的 大脑神经加工 ${ }^{[101]}$. 例如, 元分析结果表明, “看到”他人 经历疼痛会稳定激活双侧前脑岛(anterior insula, AI) 和 内侧/前扣带回(medial/anterior cingulate cortex), 这些脑 区与疼痛的情绪-动机维度加工的脑区重叠 ${ }^{[99]}$, 但共同 的脑区激活无法证明共情是“通过参与他人相同的神 经活动”实现的. 有研究者试图通过实验设计来克服这 一问题. 例如, 采用药理或心理镇痛方法能同时减弱个 体的自身疼痛和疼痛共情, 共变效应不仅体现在主观 评分上, 还体现在AI和前中扣带回(anterior medial cingulate cortex)的激活上 ${ }^{[102]}$, 而且能被阿片类药物拮抗 剂阻断 ${ }^{[101]}$, 间接证明了自身疼痛和疼痛共情涉及同样 的神经加工过程. 


\section{4 总结与展望}

\section{1 各种共情研究方法的优劣}

个体的共情能力决定了其在实际情况下产生的共 情水平的上限 ${ }^{[103]}$. 现有的共情研究方法的核心目的是 尽可能准确评估个体的共情水平，从而趋近其确切的 共情能力.

在共情研究方法中, 量表是最丰富、最具可解释性 的一种测量方式, 且便于施测. 虽然量表能够较好地反 映被试对自身共情的主观评价, 但其准确性容易受到社 会赞许、反应偏差以及被试的表达能力、觉察能力等 因素的影响. 而且, 共情量表种类繁多且缺少系统的量 表选择指南, 这给研究者选择适合的测量工具增加了难 度. 例如, IRI是目前最常用的共情自我报告量表, 很多 研究者都优先采用这一量表测量共情 ${ }^{[104]}$ ，但该量表也 是造成共情和同情概念混用的主要原因 ${ }^{[6,45]}$. 研究者对 共情及其维度的理解、测量共情的情境和目标群体都 会影响量表的选择. 而就共情行为范式而言, 研究者能 够更直接地探索特定共情情境下的被试的主观评分及 其行为表现. 通过操纵问题类型 ${ }^{[67]}$ 、评分类型 ${ }^{[69]}$ 和刺

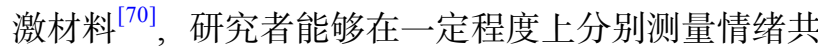
情和认知共情. 无论行为范式的形式和名称如何变化, 涉及直接的情绪分享的任务常被认为衡量的是情绪共 情, 而涉及推理他人心理状态的任务常被认为衡量的是 认知共情. 值得注意的是, 共情任务表现容易受到材料 质量、呈现视角、注意偏向等的影响. 研究者需要通过 实验设计尽可能诱发被试“真正”的共情, 例如采用多人 实验 ${ }^{101]}$ 、模拟捐款 ${ }^{[105]}$ 等方式. 与共情行为范式结合的 神经科学技术手段是主观指标的重要补充, 能够更好地 避免上述干扰因素的影响, 且能够提供被试的面部动作 模仿、脑电信号乃至大脑结构、大脑激活的客观指标, 有利于研究者深人了解共情的认知神经机制. 但是, 目 前尚无公认的“特异性”的共情生理指标, 这意味着共情 任务伴随的生理信号需要与量表、主观评分等结果相 互印证才有意义. 此外, 也有研究采集了被试在共情任 务中的自主神经系统反应，如皮肤电导、心率、呼吸以 及催产素、皮质醇等激素变化. 这些方法能够为共情的 具身表现提供丰富有效的信息,但不是共情定义的核心 内容, 故本文未详细介绍.

不同层面的共情研究方法各有所长，关联多个层 面的研究结果往往要比单一层面的证据能更好地支持 研究结论. 因此, 研究者应根据实验目的, 灵活采用主
观行为指标和客观神经科学方法. 例如, EEG的高时间 分辨率更适合探索共情的时序加工过程，即从最初的 知觉加工到情绪唤醒再到推理等高级加工过程; fMRI 的高空间分辨率更有利于研究者对共情的情绪调节、 自我他人区分、奖赏等不同功能所对应的脑区和脑网 络的探索.

\section{2 共情研究的可比性问题}

目前被不同研究采用的共情定义多达 43 个 $^{[106]}$. 由 于共情定义的不统一，不同的研究者在主观报告、行 为评分乃至客观的生理、脑机制层面可能采取不同的 研究方案并对结果进行不同的解释. 例如, 采用多个量 表测量并对比两组被试的情绪共情，可能得到不同的 评估结果 ${ }^{[107]}$; 对疼痛强度的评分可能被一个研究者解 释为情绪共情的指标，也可能被另一个研究者视为认 知共情的表现 ${ }^{[108]}$; 同样是研究疼痛共情，范式设计的 细微差异足以引起不同的脑活动 ${ }^{[90]}$. 情绪共情和认知 共情的分离也面临可比性的问题. 尽管不少研究结果 清楚地表明情绪共情和认知共情涉及不同的神经机制, 但这些研究大多依赖的是不同的刺激类型和实验任务, 难以解释两者神经机制差异的具体意义 ${ }^{[3]}$. 而且, 情绪 共情和认知共情概念上的模糊关联也使得维度分离难 以实现

多样化的探索在一定程度上有利于对共情及其机 制的宏观理解, 但也削弱了不同研究结果的可比性, 不 利于共情研究的整合和发展. 因此, 在对不同研究结果 进行比较时，研究者需特别关注其共情的定义以及采 用的测量工具和研究方法, 同类研究进行比较能更好 地避免得到错误的结论. 同时, 随着共情统一标准化定 义的呼声增加，以及对认知共情、情绪共情的心理过 程和神经机制的理解愈发精细化，共情研究结果之间 的相互印证将大大加强.

\section{3 共情研究的生态效度问题}

现有共情研究大多数采用高度简化的线索，与现 实生活情境存在一定差别. 例如, 情绪共情任务通常给 被试呈现去情景化的包含简单线索的图片，如一个浅 显的表情或动作，以诱发被试“共情”。这种共情很大程 度上受到被试的参与度或者想象力等无关变量的影响. 被试对实验材料的主观评分可能只是机械性地评价, 而没有真正“设身处地”．原本共情能力很高的个体面 对不够“生动”的材料可能无法展现真正的实力. 因此, 
研究者在今后的研究中应采用更自然的、更有生态效 度的材料和范式，如引人假被试、采用虚拟现实(virtual reality)等技术. 此外, 以往研究大多探究共情某一 子维度的加工过程及其机制, 而较少探究动态的、整
体的共情加工过程及其机制. 共情不是简单的加工过 程的叠加, 不同的共情维度存在相互作用. 因此, 今后 应该重点研究社会情境中交互的“宏观”共情的形成过 程及其原理.

\section{参考文献}

1 Chen J. Empathy for distress in humans and rodents. Neurosci Bull, 2017, 34: 216-236

2 de Waal F B M, Preston S D. Mammalian empathy: Behavioural manifestations and neural basis. Nat Rev Neurosci, 2017, 18: 498-509

3 Zaki J, Ochsner K N, Ochsner K. The neuroscience of empathy: Progress, pitfalls and promise. Nat Neurosci, 2012, 15: 675-680

4 Chen J, Shi Z B, Zhang J X. On evolvement of concept of empathy (in Chinese). Chin J Clin Psychol, 2007, 6: 664-667 [陈晶, 史占彪, 张建新. 共情概念的演变. 中国临床心理学杂志, 2007, 6: 664-667]

5 Liu C H, Wang Y M, Yu G L. Related theories and exploration on dynamic model of empathy (in Chinese). Adv Psychol Sci, 2009, 17: 964-972 [刘聪慧, 王永梅, 俞国良, 等. 共情的相关理论评述及动态模型探新. 心理科学进展, 2009, 17: 964-972]

6 Yan Z Q, Su J L, Su Y J. Empathy and sympathy or compassion: Source, conception and measurement (in Chinese). Stud Psychol Behav, 2018, 16: 433-440 [颜志强, 苏金龙, 苏彦捷. 共情与同情: 词源、概念和测量. 心理与行为研究, 2018, 16: 433-440]

7 Xiao F F, Shen H Y. On the translation and meaning of empathy (in Chinese). Psychol Explor, 2010, 30: 18-20 [肖福芳, 申荷永. 论Empathy的翻 译及其内涵. 心理学探新, 2010, 30: 18-20]

8 Mehrabian A, Epstein N. A measure of emotional empathy. J Pers, 1972, 40: 525-543

9 Mehrabian A, Young A L, Sato S. Emotional empathy and associated individual differences. Curr Psychol, 1988, 7: 221-240

10 Mehrabian A. Relations among personality scales of aggression, violence, and empathy: Validational evidence bearing on the risk of eruptive violence scale. Aggr Behav, 1997, 23: 433-445

11 Alloway $\mathrm{T}$ P, Copello $\mathrm{E}$, Loesch $\mathrm{M}$, et al. Investigating the reliability and validity of the multidimensional emotional empathy scale Measurement, 2016, 90: 438-442

12 Doherty R W. The emotional contagion scale: A measure of individual differences. J Nonv Behav, 1997, 21: 131-154

13 Hogan R. Development of an empathy scale. J Consult Clin Psychol, 1969, 33: 307-316

14 Froman R D, Peloquin S M. Rethinking the use of the Hogan empathy scale: A critical psychometric analysis. Am J Occup Ther, 2001, 55: 566572

15 Baron-Cohen S, Wheelwright S. The empathy quotient: An investigation of adults with asperger syndrome or high functioning autism, and normal sex differences. J Autism Dev Disord, 2004, 34: 163-175

16 Hojat M, Mangione S, Nasca T J, et al. The Jefferson scale of physician empathy: Development and preliminary psychometric data. Educ Psychol Measur, 2001, 61: 349-365

17 Hojat M, Lanoue M. Exploration and confirmation of the latent variable structure of the Jefferson scale of empathy. Int J Med Educ, 2014, 5: 7381

18 Hojat M. Empathy in Health Professions Education and Patient Care. New York: Springer International, 2016

19 Hojat M, Gonnella J. What matters more about the interpersonal reactivity index and the Jefferson scale of empathy? Their underlying constructs or their relationships with pertinent measures of clinical competence and patient outcomes? Acad Med, 2017, 92: 743-745

20 Davis M H. Measuring individual differences in empathy: Evidence for a multidimensional approach. J Pers Soc Psychol, 1983, 44: 113-126

21 Costa P, de Carvalho-Filho M A, Schweller M, et al. Measuring medical students' empathy. Acad Med, 2017, 92: 860-867

22 Zhan Z Y. The relations between age, sex-role, orientation of human and empathy (in Chinese). Master Thesis. Taipei: Chengchi University, 1987 [詹志禹. 年级、性别角色、人情取向与同理心的关系. 硕士学位论文. 台北: 政治大学, 1987]

23 Zhang F F, Dong Y, Wang K, et al. Reliability and validity of the Chinese version of the interpersonal reactivity index-c (in Chinese). Chin J Clin Psychol, 2010, 18: 155-157 [张凤凤, 董毅, 汪凯, 等. 中文版人际反应指针量表 (IRI-C) 的信度及效度研究. 中国临床心理学杂志, 2010, 18 155-157]

24 Cheng L, Yu X. Analysis on the reliability and validity of Chinese version of interpersonal reactivity index-c on nursing students during clinical practice (in Chinese). Mod Prevent Med, 2014, 41: 2779-2780 [程琳, 余雪. 中文版人际反应指针量表在实习护生中应用的信效度评价. 现代 预防医学, 2014, 41: 2779-2780]

25 Guan R Y, Qian M Y. Reliabilities and validities of interpersonal reactivity index among nursing students (in Chinese). Chin J Clin Psychol, 2014, 22: 493-495 [官锐园, 钱铭怡. 人际反应指数量表(IRI)应用于护理专业大学生的信度与效度分析. 中国临床心理学杂志, 2014, 22: 493495] 
26 Siu A M H, Shek D T L. Validation of the interpersonal reactivity index in a Chinese context. Res Soc Work Pract, 2005, 15: 118-126

27 Han W C, Ye M, Leng Y. Revision and examination of the interpersonal reactivity index in mainland China (in Chinese). J Southeast Univ Philos Soc Sci, 2013, 15: 47-52 [韩文超, 叶明, 冷玥. 中国大陆版人际反应指针量表的修订与检验. 东南大学学报(哲学社会科学版), 2013, 15: 47-52]

28 Rong X, Sun B H, Huang X Z, et al. Reliabilities and validities of Chinese version of interpersonal reactivity index (in Chinese). Chin J Clin Psychol, 2010, 18: 158-160 [戊幸, 孙炳海, 黄小忠, 等. 人际反应指数量表的信度和效度研究. 中国临床心理学杂志, 2010, 18: 158-160]

29 Huang X, Li W, Sun B, et al. The validation of the interpersonal reactivity index for Chinese teachers from primary and middle schools. J Psychoeduc Assess, 2012, 30: 194-204

30 Lawrence E J, Shaw P, Baker D, et al. Measuring empathy: Reliability and validity of the empathy quotient. Psychol Med, 2004, 34: 911-920

31 Zhao Q, Neumann D L, Cao X, et al. Validation of the empathy quotient in mainland China. J Pers Assess, 2018, 100: 333-342

32 Jolliffe D, Farrington D P. Development and validation of the basic empathy scale. J Adolesc, 2006, 29: 589-611

33 Geng Y, Xia D, Qin B. The basic empathy scale: A Chinese validation of a measure of empathy in adolescents. Child Psychiatry Hum Dev, 2012, 43: 499-510

34 Reniers R L E P, Corcoran R, Drake R, et al. The QCAE: A questionnaire of cognitive and affective empathy. J Pers Assess, 2011, 93: 84-95

35 Garton A F, Gringart E. The development of a scale to measure empathy in 8-and 9-year old children. Aust J Educ Dev Psychol, 2005, 5: 17-25

36 Dadds M R, Hunter K, Hawes D J, et al. A measure of cognitive and affective empathy in children using parent ratings. Child Psychiatry Hum Dev, 2008, 39: 111-122

37 Zhang Q, Wang Y, Lui S S Y, et al. Validation of the Griffith empathy measure in the Chinese context. Brain Impair, 2014, 15: 10-17

38 Baron-Cohen S, Wheelwright S, Skinner R, et al. The autism-spectrum quotient (AQ): Evidence from asperger syndrome/high-functioning autism, malesand females, scientists and mathematicians. J Autism Dev Disord, 2001, 31: 5-17

39 Caspi A, Henry B, McGee R O, et al. Temperamental origins of child and adolescent behavior problems: From age three to age fifteen. Child Dev, 1995, 66: 55-68

40 Penner L A, Fritzsche B A, Craiger J P, et al. Measuring the prosocial personality. Adv Pers Assess, 1995, 10: 147-163

41 Spreng R N, McKinnon M C, Mar R A, et al. The Toronto empathy questionnaire: Scale development and initial validation of a factor-analytic solution to multiple empathy measures. J Pers Assess, 2009, 91: 62-71

42 Wang Y W, Davidson M M, Yakushko O F, et al. The scale of ethnocultural empathy: Development, validation, and reliability. J Counsel Psychol, 2003, 50: 221-234

43 Mercer S W, Maxwell M, Heaney D, et al. The consultation and relational empathy (CARE) measure: Development and preliminary validation and reliability of an empathy-based consultation process measure. Fam Pract, 2004, 21: 699-705

44 Stern J A, Borelli J L, Smiley P A. Assessing parental empathy: A role for empathy in child attachment. Attach Hum Dev, 2015, 17: 1-22

45 Vossen H G M, Piotrowski J T, Valkenburg P M. Development of the adolescent measure of empathy and sympathy (AMES). Pers Indiv Differ, 2015, 74: 66-71

46 Rieffe C, Ketelaar L, Wiefferink C H. Assessing empathy in young children: Construction and validation of an empathy questionnaire (EMQUE) Pers Indiv Differ, 2010, 49: 362-367

47 Giummarra M J, Fitzgibbon B M, Georgiou-Karistianis N, et al. Affective, sensory and empathic sharing of another's pain: The empathy for pain scale. Eur J Pain, 2015, 19: 807-816

48 Olderbak S, Sassenrath C, Keller J, et al. An emotion-differentiated perspective on empathy with the emotion specific empathy questionnaire. Front Psychol, 2014, 5: 653

49 Ashraf S. Development and validation of the emotional empathy scale (EES) and the dispositional predictor and potential outcomes of emotional empathy. Doctor Dissertation. Islamabad: Quaid-i-Azam University, 2004

50 Rameson L T, Morelli S A, Lieberman M D. The neural correlates of empathy: Experience, automaticity, and prosocial behavior. J Cogn Neurosci, 2012, 24: $235-245$

51 Han S, Fan Y, Xu X, et al. Empathic neural responses to others' pain are modulated by emotional contexts. Hum Brain Mapp, 2009, 30: 32273237

52 Jackson P L, Meltzoff A N, Decety J. How do we perceive the pain of others? A window into the neural processes involved in empathy. NeuroImage, 2005, 24: 771-779

53 Barraza J A, Zak P J. Empathy toward strangers triggers oxytocin release and subsequent generosity. Ann New York Acad Sci, 2009, 1167: 182189

54 Singer T, Seymour B, O’Doherty J, et al. Empathy for pain involves the affective but not sensory components of pain. Science, 2004, 303: 11571162

55 Greitemeyer T, Osswald S, Brauer M. Playing prosocial video games increases empathy and decreases schadenfreude. Emotion, 2010, 10: 796- 
802

56 Cheng Y, Chen C, Lin C P, et al. Love hurts: An fMRI study. NeuroImage, 2010, 51: 923-929

57 Jackson P L, Brunet E, Meltzoff A N, et al. Empathy examined through the neural mechanisms involved in imagining how I feel versus how you feel pain. Neuropsychologia, 2006, 44: 752-761

58 Saarela M V, Hlushchuk Y, Williams A C C, et al. The compassionate brain: Humans detect intensity of pain from another's face. Cereb Cortex, 2006, 17: 230-237

59 Shamay-Tsoory S G, Aharon-Peretz J, Perry D. Two systems for empathy: A double dissociation between emotional and cognitive empathy in inferior frontal gyrus versus ventromedial prefrontal lesions. Brain, 2009, 132: 617-627

$60 \mathrm{Gu}$ X, Liu X, Guise K G, et al. Functional dissociation of the frontoinsular and anterior cingulate cortices in empathy for pain. J Neurosci, 2010, 30: 3739-3744

61 Coll M P, Viding E, Rütgen M, et al. Are we really measuring empathy? Proposal for a new measurement framework. Neurosci Biobehaval Rev, 2017, 83: 132-139

62 Kanske P, Böckler A, Trautwein F M, et al. Dissecting the social brain: Introducing the empatom to reveal distinct neural networks and brainbehavior relations for empathy and theory of mind. NeuroImage, 2015, 122: 6-19

63 Schnell K, Bluschke S, Konradt B, et al. Functional relations of empathy and mentalizing: An fMRI study on the neural basis of cognitive empathy. NeuroImage, 2011, 54: 1743-1754

64 Jones A P, Happé F G E, Gilbert F, et al. Feeling, caring, knowing: Different types of empathy deficit in boys with psychopathic tendencies and autism spectrum disorder. J Child Psychol Psychiatry, 2010, 51: 1188-1197

65 Walter H. Social cognitive neuroscience of empathy: Concepts, circuits, and genes. Emot Rev, 2012, 4: 9-17

66 Shamay-Tsoory S G, Aharon-Peretz J. Dissociable prefrontal networks for cognitive and affective theory of mind: A lesion study. Neuropsychologia, 2007, 45: 3054-3067

67 Dziobek I, Rogers K, Fleck S, et al. Dissociation of cognitive and emotional empathy in adults with asperger syndrome using the multifaceted empathy test (MET). J Autism Dev Disord, 2008, 38: 464-473

68 Dziobek I, Preissler S, Grozdanovic Z, et al. Neuronal correlates of altered empathy and social cognition in borderline personality disorder. NeuroImage, 2011, 57: 539-548

69 Mischkowski D, Crocker J, Way B M. From painkiller to empathy killer: Acetaminophen (paracetamol) reduces empathy for pain. Soc Cogn Affect Neurosci, 2016, 11: 1345-1353

70 Nummenmaa L, Hirvonen J, Parkkola R, et al. Is emotional contagion special? An fMRI study on neural systems for affective and cognitive empathy. NeuroImage, 2008, 43: 571-580

71 Dimberg U, Thunberg M, Elmehed K. Unconscious facial reactions to emotional facial expressions. Psychol Sci, 2000, 11: 86-89

72 Mancini G, Ferrari P F, Palagi E. Rapid facial mimicry in geladas. Sci Rep, 2013, 3: 1527

73 Neumann D L, Westbury H R. The psychophysiological measurement of empathy. In: Scapaletti D J, ed. Psychology of Empathy. New York: Nova Science Publishers Inc, 2011. 119-142

74 Neumann D L, Chan R C, Boyle G J, et al. Measures of empathy: Self-report, behavioral, and neuroscientific approaches. In: Boyle G J, Saklofske D H, Matthews G, eds. Measures of Personality and Social Psychological Constructs. London: Academic Press, 2014. 257-289

75 Dimberg U, Thunberg M. Empathy, emotional contagion, and rapid facial reactions to angry and happy facial expressions. Psychol J, 2012, 1: $118-127$

76 Dimberg U, Andréasson P, Thunberg M. Emotional empathy and facial reactions to facial expressions. J Psychophysiol, 2011, 25: 26-31

77 Rae Westbury H, Neumann D L. Empathy-related responses to moving film stimuli depicting human and non-human animal targets in negative circumstances. Biol Psychol, 2008, 78: 66-74

78 Cheng Y, Chen C, Decety J. An EEG/ERP investigation of the development of empathy in early and middle childhood. Dev Cogn Neurosci, 2014, 10: $160-169$

79 Fan Y, Han S. Temporal dynamic of neural mechanisms involved in empathy for pain: An event-related brain potential study. Neuropsychologia, 2008, 46: 160-173

80 Vaes J, Meconi F, Sessa P, et al. Minimal humanity cues induce neural empathic reactions towards non-human entities. Neuropsychologia, 2016, 89: $132-140$

81 Fabi S, Leuthold H. Empathy for pain influences perceptual and motor processing: Evidence from response force, ERPs, and EEG oscillations. Soc Neurosci, 2017, 12: 701-716

82 Meng J, Hu L, Shen L, et al. Emotional primes modulate the responses to others' pain: An ERP study. Exp Brain Res, 2012, 220: 277-286

83 Cheng Y, Hung A Y, Decety J. Dissociation between affective sharing and emotion understanding in juvenile psychopaths. Dev Psychopathol, 2012, 24: 623-636 
84 Cui F, Zhu X, Luo Y. Social contexts modulate neural responses in the processing of others' pain: An event-related potential study. Cogn Affect Behav Neurosci, 2017, 17: 850-857

85 Ikezawa S, Corbera S, Wexler B E. Emotion self-regulation and empathy depend upon longer stimulus exposure. Soc Cogn Affect Neurosci, 2013, 9: $1561-1568$

86 Suzuki Y, Galli L, Ikeda A, et al. Measuring empathy for human and robot hand pain using electroencephalography. Sci Rep, 2015, 5: 15924

87 Sheng F, Han S. Manipulations of cognitive strategies and intergroup relationships reduce the racial bias in empathic neural responses. NeuroImage, 2012, 61: 786-797

88 Decety J, Yang C Y, Cheng Y. Physicians down-regulate their pain empathy response: An event-related brain potential study. NeuroImage, 2010, 50: $1676-1682$

89 Li W, Han S. Perspective taking modulates event-related potentials to perceived pain. NeuroSci Lett, 2010, 469: 328-332

90 Coll M P. Meta-analysis of ERP investigations of pain empathy underlines methodological issues in ERP research. Soc Cogn Affect Neurosci, 2018, 13: 1003-1017

91 Schupp H T, Junghöfer M, Weike A I, et al. Attention and emotion: An ERP analysis of facilitated emotional stimulus processing. NeuroReport, 2003, 14: 1107-1110

92 Yang C Y, Decety J, Lee S, et al. Gender differences in the mu rhythm during empathy for pain: An electroencephalographic study. Brain Res, 2009, 1251: 176-184

93 Perry A, Bentin S, Bartal I B A, et al. "Feeling" the pain of those who are different from us: Modulation of EEG in the mu/alpha range. Cogn Affect Behav Neurosci, 2010, 10: 493-504

94 Davidson R J. What does the prefrontal cortex "do" in affect: Perspectives on frontal EEG asymmetry research. Biol Psychol, 2004, 67: 219-234

95 Gutsell J N, Inzlicht M. Intergroup differences in the sharing of emotive states: Neural evidence of an empathy gap. Soc Cogn Affect Neurosci, 2012, 7: 596-603

96 Tullett A M, Harmon-Jones E, Inzlicht M. Right frontal cortical asymmetry predicts empathic reactions: Support for a link between withdrawal motivation and empathy. Psychophysiology, 2012, 49: 1145-1153

97 Levy J, Goldstein A, Pratt M, et al. Maturation of pain empathy from child to adult shifts from single to multiple neural rhythms to support interoceptive representations. Sci Rep, 2018, 8: 1810

98 Banissy M J, Kanai R, Walsh V, et al. Inter-individual differences in empathy are reflected in human brain structure. NeuroImage, 2012, 62: 2034-2039

99 Lamm C, Decety J, Singer T. Meta-analytic evidence for common and distinct neural networks associated with directly experienced pain and empathy for pain. NeuroImage, 2011, 54: 2492-2502

$100 \mathrm{Hu}$ L, Iannetti G D. Painful issues in pain prediction. Trends Neurosci, 2016, 39: 212-220

101 Rütgen M, Seidel E M, Silani G, et al. Placebo analgesia and its opioidergic regulation suggest that empathy for pain is grounded in self pain. Proc Natl Acad Sci USA, 2015, 112: E5638-E5646

102 Rütgen M, Seidel E M, Riečanský I, et al. Reduction of empathy for pain by placebo analgesia suggests functional equivalence of empathy and first-hand emotion experience. J Neurosci, 2015, 35: 8938-8947

103 Keysers C, Gazzola V. Dissociating the ability and propensity for empathy. Trends Cogn Sci, 2014, 18: $163-166$

104 Yan Z Q, Su Y J. Evolution in research topics on empathy: Evidence from bibliometrics (in Chinese). Psychol Sci, 2017, 40: 699-707 [颜志强, 苏彦捷. 共情研究主题的变化——来自文献计量学的证据. 心理科学, 2017, 40: 699-707]

105 Cameron C D, Spring V L, Todd A R. The empathy impulse: A multinomial model of intentional and unintentional empathy for pain. Emotion, 2017, 17: 395-411

106 Cuff B M P, Brown S J, Taylor L, et al. Empathy: A review of the concept. Emot Rev, 2016, 8: 144-153

107 Peretti S, Tempesta D, Socci V, et al. The role of sleep in aesthetic perception and empathy: A mediation analysis. J Sleep Res, 2018, 32: e12664

108 Lamm C, Nusbaum H C, Meltzoff A N, et al. What are you feeling? Using functional magnetic resonance imaging to assess the modulation of sensory and affective responses during empathy for pain. PLoS One, 2007, 2: e1292 


\title{
Empathy: Methodologies and characteristics from a psychophysiological perspective
}

\author{
Qiaoyue Ren ${ }^{1,2}$, Yuanmiao $\mathrm{Sun}^{3}$, Xuejing $\mathrm{Lü}^{1,2^{*}}$, Chao Huang ${ }^{4} \& \mathrm{Li} \mathrm{Hu}^{1,2^{*}}$ \\ ${ }^{1}$ Key Laboratory of Mental Health, Institute of Psychology, Chinese Academy of Sciences, Beijing 100101, China; \\ 2 Department of Psychology, University of Chinese Academy of Sciences, Beijing 100049, China; \\ ${ }^{3}$ Research Center of Brain Cognitive Neuroscience, Liaoning Normal University, Dalian 116029, China; \\ ${ }^{4}$ Department of Medical Psychology, School of Medical Humanities, Capital Medical University, Beijing 100069, China \\ * Corresponding authors, E-mail: luxj@psych.ac.cn; huli@psych.ac.cn
}

Empathy is an essential skill in our social lives, allowing us to understand and share others' feelings. In the last decade, empathy has been a hot topic in cognitive neuroscience for its important role in social interaction. To date, there are a variety of definitions of the term "empathy", such as "a reaction to the observed experiences of another" and "the ability to experience and understand what others feel without confusion between oneself and others". Based on different definitions of empathy, different methodologies have been developed and adopted in the field of empathy research. Valid empathy questionnaires are developed to provide ones' subjective evaluation about their empathy ability (i.e., trait empathy); behavioral paradigms are designed to measure individuals' empathy levels under a specific context (i.e., state empathy); physiological techniques and brain imaging techniques are useful in detecting objective physiological signals related to empathy. However, some findings on empathy are mixed and inconsistent, which might be due to the different definitions and methodologies adopted by each study. To deepen our understanding of empathy and its psychological mechanisms, it is necessary to integrate different methodologies and interpret results from different aspects. To this end, this review first summarized how empathy is typically measured and quantified at different measurement levels with an emphasis on emotional empathy and cognitive empathy, given that existing studies on empathy mainly focus on the two categories and their combination. Specifically, methodologies in empathy research were introduced as followed: (1) Widely-used empathy questionnaires, including the detailed definitions and measurement dimensions; (2) classic experimental materials and behavioral paradigms for measuring emotional empathy and/or cognitive empathy; (3) empathy-related physiological signals detected by facial electromyography, electroencephalography (EEG), magnetoencephalography (MEG), and magnetic resonance imaging (MRI). Next, we discussed the advantages and disadvantages of each methodology and pointed out the difficulties in compiling findings from studies using different methodologies. For example, different empathy questionnaires applied to the same group may yield contradictory results and the quality of experimental stimulus in the same kind of empathy paradigms potentially influences participants' brain activities. More importantly, the physiological signals (e.g. event-related potential components) recorded in empathy experiments are not "specific" but only "related" to empathy. Then, we suggested that researchers should carefully select appropriate methodologies according to their research needs. For instance, EEG is suitable for exploring empathic processing in the time domain, while functional magnetic resonance imaging (fMRI) is conducive to pinpoint the specific functions (e.g. self-other discrimination) of empathy-related brain regions. Finally, we proposed that the conceptual framework of empathy should be unified and more naturalistic paradigms should be developed to reveal the interactions among different empathic subprocesses in the future. In conclusion, this review highlights the importance of comparing and combining different methodologies to reveal the underlying mechanisms of empathy, which yields new insights into social interaction.

empathy, emotional empathy, cognitive empathy, questionnaire, behavioral paradigm, physiological signal

doi: 10.1360/N972019-00276 